\title{
Secagem drástica de madeiras de Eucalyptus e Corymbia fornece informações para a elaboração de programas de secagem
}

\author{
Jackson Roberto Eleotério ${ }^{1 *}$, Tania Regina Bagattoli ${ }^{1}$, Kerling Fabiane Hornburg ${ }^{1}$, Cláudia Mariana Kirchheim da Silva ${ }^{1}$ \\ ${ }^{1}$ Universidade Regional de Blumenau, Rua São Paulo, 3250, CEP 89030-000, Blumenau, SC, Brasil
}

*Autor correspondente:

jreleote@furb.br

Termos para indexação:

Secagem convencional

Rachaduras

Taxa de secagem

Index terms:

Conventional drying

Cracks

Drying taxes

Histórico do artigo:

Recebido em 24/04/2014

Aprovado em 10/12/2015

Publicado em 31/12/2015

doi: $10.4336 / 2015 . p f b .35 .84 .696$

\begin{abstract}
Resumo - O objetivo deste trabalho foi avaliar a secagem drástica de pequenas amostras como alternativa metodológica para a definição de programas de secagem umidadetemperatura, aplicando-a para madeira de Corymbia torelliana, Eucalyptus cloeziana, E. grandis x E. urophylla, E. pilularis e E. resinifera e avaliar a possibilidade de agrupar espécies com recomendações semelhantes. Foram utilizadas peças com dimensões iniciais de $100 \mathrm{~mm}$ de comprimento, $50 \mathrm{~mm}$ de largura e $10 \mathrm{~mm}$ de espessura. Essas foram mantidas em estufa a $100{ }^{\circ} \mathrm{C}$, até que o teor de umidade atingisse valores inferiores a $5 \%$. Durante a secagem drástica as peças foram pesadas em intervalos de $1 \mathrm{~h}$ e foram medidos o comprimento e a largura das rachaduras, utilizando paquímetro e calibrador de folga. Em função das diferenças nas temperaturas iniciais, finais e no potencial de secagem, observou-se um grupo que suporta condições de secagem mais severas, formado por Corymbia torelliana e E. grandis x E. urophylla e outro que demanda condições mais suaves, formado por E. pilularis e E. cloeziana. A madeira de $E$. resinifera pode ser submetida à secagem nas condições definidas para quaisquer dos grupos.
\end{abstract}

\section{Drastic drying on Eucalyptus and Corymbia wood provides information to the dry kiln schedules elaboration}

\begin{abstract}
The aim of this work was to evaluate drastic drying of small pieces as alternative method to define moisture content-temperature dry kiln schedules, to be applied on wood of Corymbia torelliana, Eucalyptus cloeziana, E. grandis x E. urophylla, E. pilularis and E. resinifera. Moreover, it was aimed to evaluate the possibility to group different species with similar recommended conditions. It was used pieces with initial dimensions of $100 \mathrm{~mm}$ long, $50 \mathrm{~mm}$ wide and $10 \mathrm{~mm}$ thick, maintained in kiln at $100^{\circ} \mathrm{C}$ until moisture content reached values below $5 \%$. During this drastic drying the pieces were weighed every hour and length and width of cracks were measure with caliper and feeler gauges. Considering initial and final temperature and drying potential, it was observed one group that supports more severe drying conditions, formed by Corymbia torelliana and E. grandis $\mathrm{x}$ E. urophylla and another that demands conservative conditions formed by E. pilularis and E. cloeziana. E. resinifera wood can be dried at conditions defined to both groups.
\end{abstract}

\section{Introdução}

Embora o gênero Eucalyptus represente uma alternativa potencial para o abastecimento da indústria madeireira, sua madeira apresenta restrições próprias e inerentes ao uso de florestas jovens, em que os níveis de tensões de crescimento se manifestam de forma mais proeminente do que em florestas maduras (Assis, 1999). O mesmo autor considera que as perdas 
significativas em função das rachaduras associadas às tensões de crescimento e aos defeitos de secagem têm sido consideradas dentre os principais entraves à utilização econômica de espécies de Eucalyptus. Apesar da consolidada utilização da madeira de eucalipto como matéria-prima nas indústrias de celulose, carvão e painéis de madeira reconstituída, o mesmo não ocorre em larga escala com a indústria de madeira serrada (Longue Júnior \& Colodette, 2013; Colodette et al.; 2014).

A madeira de E. grandis não apresenta a fase de taxa constante na sua curva característica de secagem, indicando a impermeabilidade (Jankowsky et al., 2003). A impermeabilidade implica na necessidade de secagem lenta e no desenvolvimento, durante o processo, de gradientes de umidade acima do normal. As baixas taxas de secagem são atribuídas por Redman et al. (2012) às difusividades comparativamente baixas das madeiras australianas.

De acordo com Ducatti et al. (2001), assegurar um padrão elevado de qualidade implica necessariamente em preparar adequadamente a madeira e controlar o processo de forma a manter as condições do meio de secagem compatíveis com as características do material. $\mathrm{O}$ equilíbrio entre qualidade da madeira seca e produtividade do sistema de secagem constitui um dos maiores desafios para a definição de um programa de secagem.

Os programas de secagem podem ser definidos como uma sequência pré-estabelecida, contendo alterações adequadas de temperatura e umidade relativa do ar, que deve ser aplicada a uma carga de madeira durante a secagem (Galvão \& Jankowsky, 1985). Em geral, a madeira de eucalipto deve ser seca em condições suaves de temperatura e umidade relativa do ar, proporcionando uma secagem mais lenta e com menor quantidade de defeitos (Eucalipto..., 2001).

Os principais fatores envolvidos na escolha do programa de secagem são: espécie de madeira, teor de umidade inicial e final, espessura do material, uso a que se destina e equipamento de secagem (Martins, 1988). Simpson (1996) apresentou uma sequência de passos para determinar o programa de secagem baseado na massa específica, porém o método não é aplicável à madeira de Eucalyptus devido à tendência anormal de formação de colapso. Por outro lado, a secagem drástica foi utilizada por Andrade (2000), Santos (2002) e Jankowsky (2009) para o estabelecimento de programas de secagem para Eucalyptus. Estes autores obtiveram programas de secagem com as características recomendadas de baixa temperatura e baixo potencial de secagem. A secagem drástica também foi utilizada para estimar programas de secagem para híbridos naturais de Eucalyptus (Barbosa et al., 2005) e para espécies tropicais (Brandão \& Jankowsky, 1992; Eleotério \& Silva, 2012, 2014).

O objetivo deste trabalho foi avaliar a secagem drástica de pequenas amostras como alternativa metodológica para a definição de programas de secagem umidade-temperatura, para uso em madeira de Corymbia torelliana, E. cloeziana, E. grandis x E. urophylla, E. pilularis e E. resinifera e avaliar a possibilidade de agrupar diferentes espécies com recomendações de secagem semelhantes.

\section{Material e métodos}

As amostras de madeira de Corymbia torelliana F. Muell., E. cloeziana F. Muell., E. resinifera Sm. e E. pilularis $\mathrm{Sm}$. foram obtidas de um plantio experimental com 17 anos, localizado no município de Garuva, SC e as do híbrido E. grandis W Hill ex Maiden x E. urophylla S. T. Blake de um plantio com oito anos localizado em Barra Velha, SC.

O método da secagem drástica se baseia no princípio de que pequenas amostras de madeira, quando expostas aos efeitos de secagem drástica em estufa a $100{ }^{\circ} \mathrm{C}$, proporcionam um comportamento semelhante à secagem convencional. O método foi baseado nas recomendações e metodologias apresentadas em Jankowsky (2009).

Para a obtenção do teor de umidade inicial, da massa específica e dos parâmetros necessários para a determinação do programa de secagem, foram utilizadas 17 amostras de madeira de E. cloeziana; 16 de E. pilularis e de E. grandis x E. urophylla, 15 de Corymbia torelliana e 12 de E. resinifera, com dimensões iniciais de $100 \mathrm{~mm}$ de comprimento, $50 \mathrm{~mm}$ de largura e 10 $\mathrm{mm}$ de espessura. Estas amostras foram mantidas em estufa a $100{ }^{\circ} \mathrm{C}$, até que atingisse valores inferiores a $5 \%$ de umidade. Durante a secagem drástica, as amostras foram pesadas em intervalos de $1 \mathrm{~h}$, sendo medidos o comprimento e a largura das rachaduras, utilizando paquímetro e calibrador de folga. A magnitude das rachaduras foi transformada em escores de acordo com Jankowsky (2009).

Após finalizada a etapa de determinação das taxas de secagem e das dimensões das rachaduras, as amostras 
foram transferidas para uma estufa com ventilação forçada, regulada com temperatura de $103 \pm 2{ }^{\circ} \mathrm{C}$, até estabilização da massa (considerada a massa seca). A massa específica básica por peça foi determinada como a relação entre a massa seca e o volume inicial, determinado pelo método estereométrico. $\mathrm{O}$ teor de umidade ao longo da secagem foi determinado pela relação entre a massa de água e a massa seca. A massa de água foi determinada pela diferença entre a massa aparente e a massa seca.

Os parâmetros para a definição dos programas de secagem: temperatura inicial (Ti), temperatura final (Tf) e potencial de secagem (PS) foram obtidos através das taxas e velocidades de secagem e dos escores obtidos através das dimensões dos defeitos. As equações 1 a 3, extraídas de Jankowsky (2009), estimam os parâmetros do programa de secagem em função das taxas, velocidades e escores obtidos na secagem drástica.

$\mathrm{T}_{\mathrm{i}}=27,9049+0,7881\left(\mathrm{~T}_{2}\right)+419,0254\left(\mathrm{~V}_{1}\right)+1,9483\left(\mathrm{R}_{2}\right)$

$\mathrm{T}_{\mathrm{f}}=49,2292+1,1834\left(\mathrm{~T}_{2}\right)+273,8685\left(\mathrm{~V}_{2}\right)+1,0754\left(\mathrm{R}_{1}\right)$
PS $=1,4586-30,4418\left(\mathrm{~V}_{3}\right)+42,9653\left(\mathrm{~V}_{1}\right)+0,1424\left(\mathrm{R}_{3}\right)$

Onde:

$\mathrm{T}_{2}=$ tempo de secagem da umidade inicial até $30 \%(\mathrm{~h}) ;$

$\mathrm{V}_{1}=$ taxa de secagem da umidade inicial até $5 \%\left(\mathrm{~g} \mathrm{~cm}^{-2} . \mathrm{h}\right)$;

$\mathrm{V}_{2}=$ taxa de secagem da umidade inicial até $30 \%$ $\left(\mathrm{g} \mathrm{cm}^{-2} \cdot \mathrm{h}^{-1}\right)$;

$\mathrm{V}_{3}=$ taxa de secagem de 30 a $5 \%$ de umidade $\left(\mathrm{g} \mathrm{cm}^{-2} \cdot \mathrm{h}^{-1}\right)$;

$\mathrm{R}_{1}=$ intensidade das rachaduras da umidade inicial até $5 \%$;

$\mathrm{R}_{2}=$ intensidade das rachaduras da umidade inicial até $30 \%$;

$\mathrm{R}_{3}=$ intensidade das rachaduras de $30 \%$ a $5 \%$.

A apresentação detalhada da metodologia é encontrada em Jankowsky (2009). Os parâmetros dos programas de secagem foram comparados entre espécies pelo teste de Tukey com $\alpha=5 \%$.

\section{Resultados e discussão}

Os valores médios obtidos por secagem drástica, bem como umidade inicial, massa específica básica e demais variáveis utilizadas para a determinação dos parâmetros do programa de secagem estão apresentados na Tabela 1 .

Tabela 1. Valores médios do teor de umidade inicial $\left(\mathrm{U}_{\mathrm{i}}\right)$, massa específica básica $\left(\mathrm{r}_{\text {bás }}\right)$, tempo de secagem até $30 \%$ de umidade $\left(T_{2}\right)$, fluxo de umidade $\left(V_{1}\right.$ a $\left.V_{3}\right)$ e escores de defeitos $\left(R_{1}\right.$ a $\left.R_{3}\right)$.

\begin{tabular}{|c|c|c|c|c|c|c|c|c|c|}
\hline \multirow[t]{2}{*}{ Espécie } & $\mathbf{U i}$ & $\underset{\left(\mathrm{g} \mathrm{cm}^{-3}\right)}{\rho_{\text {bas }}}$ & $\mathbf{T}_{2}$ & $\mathbf{V}_{1}$ & $\mathbf{V}_{2}$ & $\mathbf{V}_{3}$ & \multirow[t]{2}{*}{$\mathbf{R}_{1}$} & \multirow[t]{2}{*}{$\mathbf{R}_{2}$} & \multirow[t]{2}{*}{$\mathbf{R}_{3}$} \\
\hline & $\%$ & & (h) & \multicolumn{3}{|c|}{$\left(\mathrm{g} \mathrm{cm}^{-2} \cdot \mathrm{h}^{-1}\right)$} & & & \\
\hline Corymbia torelliana & 78,6 & 0,65 & 3,0 & 0,0278 & 0,0536 & 0,0150 & 1,53 & 1,53 & 1,47 \\
\hline Eucalyptus cloeziana & 68,8 & 0,58 & 2,0 & 0,0199 & 0,0559 & 0,0104 & 1,00 & 1,00 & 1,00 \\
\hline E. grandis $x$ E. urophylla & 109,0 & 0,46 & 2,7 & 0,0344 & 0,0733 & 0,0144 & 1,00 & 1,00 & 1,00 \\
\hline E. pilularis & 134,7 & 0,40 & 5,6 & 0,0220 & 0,0371 & 0,0092 & 1,00 & 1,00 & 1,00 \\
\hline E. resinifera & 84,1 & 0,61 & 2,6 & 0,0251 & 0,0546 & 0,0116 & 1,50 & 1,50 & 1,50 \\
\hline
\end{tabular}

As espécies de eucaliptos apresentaram massas específicas que permitem enquadrá-las, de acordo com os critérios estabelecidos por Durlo (1991), como madeiras leves e médias, sendo E. pilularis e o hibrído $E$. grandis $\mathrm{x}$ E. urophylla consideradas leves, E. cloeziana, E. resinifera madeiras médias, e, por fim, Corymbia torelliana foi a mais pesada dentre as estudadas.

A caracterização das condições iniciais das amostras confirma a relação inversa entre a massa específica básica e o teor de umidade inicial. Uma correlação significativa de $-82 \%$ foi obtida entre estas variáveis. A relação exponencial negativa entre a massa específica básica e o teor de umidade inicial também foi apresentada por Simpson (1996), sendo aplicadas as equações apresentadas por este autor e comparadas com os dados obtidos no trabalho atual (Figura 1). Apesar da variabilidade entre peças, observa-se que a estimativa representa a média dos dados experimentais.

O teor de umidade inicial é um dos parâmetros utilizados por Gorvard \& Arganbright (1979) para calcular um índice para expressar a intensidade de um programa de secagem. Esse parâmetro relaciona-se com a ocorrência de defeitos como o colapso, muito comum na madeira de eucalipto. Além do teor de umidade inicial, a taxa de secagem, expressa pela variação no teor de umidade, desde a inicial até a final, em função do tempo também é um componente desse índice de severidade. 


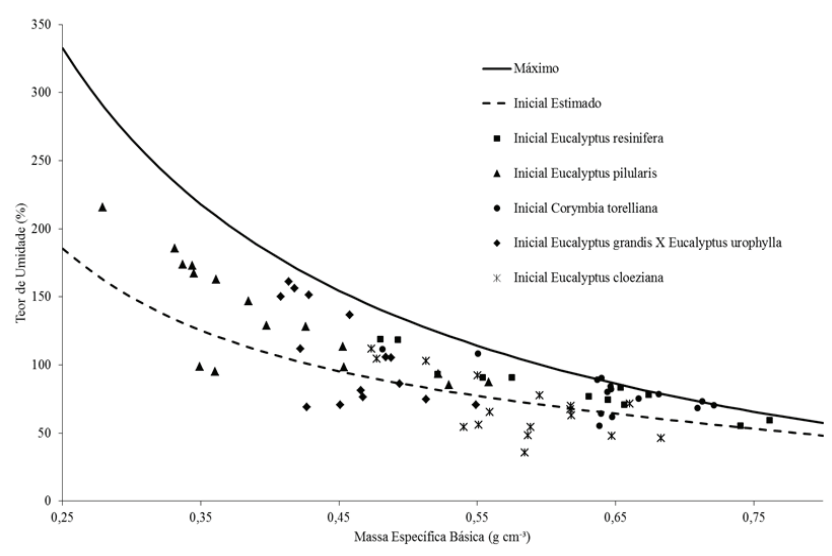

Figura 1. Teor de umidade inicial em função da massa específica básica.

Os valores encontrados para as massas específicas da madeira de E. cloeziana e de E. pilularis foram menores e os teores de umidade iniciais maiores (Tabela 1) do que encontrado em literatura. Andrade (2000) encontrou, para $E$. grandis, massa específica básica de $0,54 \mathrm{~g} \mathrm{~cm}^{-3}$ e umidade inicial de $84,5 \%$ e para E. tereticornis massa específica básica de $0,80 \mathrm{~g} \mathrm{~cm}^{-3}$ e umidade de $48,3 \%$. Oliveira et al. (2005) encontraram para madeira de Corymbia citriodora, massa específica básica de 0,73 $\mathrm{g} \mathrm{cm}^{-3}$ com umidade inicial de $62 \%$ e para $E$. grandis, massa específica de $0,49 \mathrm{~g} \mathrm{~cm}^{-3}$ e umidade inicial com $102 \%$, para E. cloeziana e E. pilularis, massa específica de 0,72 e $0,60 \mathrm{~g} \mathrm{~cm}^{-3}$, respectivamente, e umidade inicial de $63,9 \%$ e $86,3 \%$, respectivamente. Santos (2002) obteve para madeira de E. grandis, resultados superiores com relação ao tempo, de 4,01 h, e inferiores para a velocidade de secagem, de $0,017 \mathrm{~h} ; 0,0335 \mathrm{~h}$ e 0,0091 h para V1, V2 e V3 respectivamente, aos obtidos nesse trabalho (Tabela 1), entretanto, os valores de rachadura foram similares.

Para a indicação dos programas de secagem, a massa específica básica não deve ser o único fator a ser considerado. Andrade (2000) descreve que esse fator não pode ser analisado isoladamente, pois algumas espécies apresentam massa específica básica semelhante. Porém, a velocidade de secagem e a ocorrência de defeitos têm variação entre as espécies, devido a diferenças na estrutura anatômica da madeira.

As variáveis apresentadas na Tabela 1 foram utilizadas nas equações 1 a 3 para definir os parâmetros fundamentais dos programas de secagem, que são apresentados na Tabela 2.
Tabela 2. Temperatura inicial, temperatura final e potencial de secagem para a madeira das espécies de eucalipto.

\begin{tabular}{lccc}
\hline \multicolumn{1}{c}{ Espécie } & $\begin{array}{c}\text { Temperatura } \\
\text { inicial }\left({ }^{\circ} \mathbf{C}\right)\end{array}$ & $\begin{array}{c}\text { Temperatura } \\
\text { final }\left({ }^{\circ} \mathbf{C}\right)\end{array}$ & $\begin{array}{c}\text { Potencial de } \\
\text { secagem }\end{array}$ \\
\hline Corymbia torelliana & $47,3 \mathrm{a}$ & $70,8 \mathrm{ab}$ & $2,44 \mathrm{~b}$ \\
Eucalyptus cloeziana & $40,0 \mathrm{~b}$ & $68,7 \mathrm{~b}$ & $2,16 \mathrm{~b}$ \\
E. grandis $x$ E. urophylla & $46,5 \mathrm{a}$ & $73,6 \mathrm{a}$ & $2,66 \mathrm{a}$ \\
E. pilularis & $43,1 \mathrm{ab}$ & $66,5 \mathrm{~b}$ & $2,24 \mathrm{ab}$ \\
E. resinifera & $45,0 \mathrm{ab}$ & $70,1 \mathrm{ab}$ & $2,49 \mathrm{ab}$ \\
\hline
\end{tabular}

Médias na coluna seguidas pela mesma letra não diferem entre si pelo teste de Tukey ao nível de $5 \%$ de probabilidade.

Analisando-se conjuntamente os três parâmetros do programa de secagem apresentados na Tabela 2, pode-se observar dois grupos: um deles formado por Corymbia torelliana e E. grandis x E. urophylla e o outro por E. pilularis e E. cloeziana. E. resinifera pode integrar qualquer dos dois grupos. Para o primeiro grupo são recomendadas temperaturas e potenciais de secagem maiores que para o segundo grupo, sendo que as temperaturas iniciais variaram de 40 a $47,3^{\circ} \mathrm{C}$ e as temperaturas finais ficaram entre 66,5 e $73,6{ }^{\circ} \mathrm{C}$. Para analisar a severidades desses programas é necessário aplicá-los e avaliar a variação nos defeitos entre o início e o final da secagem (Eleotério et al., 2014). Programas de secagem para espécies com baixa difusividade e alta retratibilidade podem ser severos, mesmo em condições de baixas temperaturas e altas umidades relativas do ar. Severo (2000) observou em E. dunnii a formação de colapso e de encanoamento em $37,5 \%$ e $35 \%$ das peças, respectivamente, mesmo quando a madeira foi seca com temperatura de bulbo seco que não ultrapassou $45^{\circ} \mathrm{C}$ até o ponto de saturação das fibras. Para a mesma espécie, Batista et al. (2015) registraram a formação de colapso, encanoamento e de tensões de secagem fortes em 55,6\%, $74,1 \%$ e $59,3 \%$ das peças, respectivamente, mesmo utilizando um programa de secagem com temperatura igual ou inferior a $46{ }^{\circ} \mathrm{C}$ e umidade relativa do ar igual ou superior a $78 \%$ até que a madeira atingisse teor de umidade médio de $31 \%$.

As temperaturas médias inicial e final e o potencial de secagem médio apresentados por Barbosa et al. (2005) estão contidos no intervalo determinado nesse trabalho (Tabela 2). 
Foram verificadas correlações fracas entre massa específica e temperatura inicial, temperatura final e potencial de secagem, de $16,1 \%, 22,1 \%$ e $3,2 \%$, respectivamente, o que reforça a ressalva apresentada por Simpson \& Verrill (1997) de que a estimativa de programas de secagem com base apenas na massa específica é um método imperfeito e que pode resultar em desvios consideráveis do ideal. Apesar de apresentar um método para determinar programas de secagem com base na massa específica, Simpson (1996) não recomenda a aplicação do método para eucaliptos.

Os programas de secagem para cada espécie são apresentados na Tabela 3 e foram elaborados com os parâmetros apresentados na Tabela 2. Não estão incluídas as fases de uniformização e condicionamento, pois estas dependem do teor de umidade final desejado.

Tabela 3. Programas de secagem umidade-temperatura para diferentes espécies de eucalipto.

\begin{tabular}{|c|c|c|c|c|c|c|c|c|c|c|}
\hline \multirow[t]{2}{*}{$\begin{array}{l}\text { Umidade da } \\
\text { madeira }(\%)\end{array}$} & \multicolumn{2}{|c|}{ 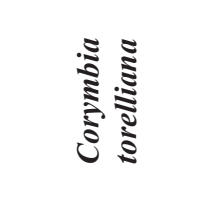 } & \multicolumn{2}{|c|}{ 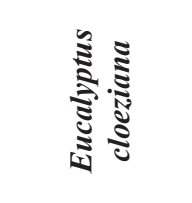 } & \multicolumn{2}{|c|}{ 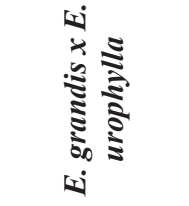 } & \multicolumn{2}{|c|}{ 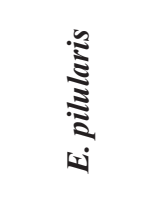 } & \multicolumn{2}{|c|}{ 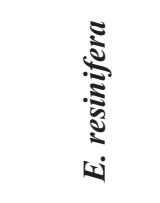 } \\
\hline & TBS & PS & TBS & PS & TBS & PS & TBS & PS & TBS & PS \\
\hline $50-45$ & 47,3 & 3,3 & 40,0 & 3,3 & 46,5 & 3,3 & 43,1 & 3,3 & 45,0 & 3,3 \\
\hline $45-40$ & 47,3 & 3,1 & 40,0 & 3,0 & 46,5 & 3,1 & 43,1 & 3,0 & 45,0 & 3,1 \\
\hline $40-35$ & 47,3 & 2,9 & 40,0 & 2,7 & 46,5 & 3,0 & 43,1 & 2,8 & 45,0 & 2,9 \\
\hline $35-30$ & 47,3 & 2,7 & 40,0 & 2,4 & 46,5 & 2,8 & 43,1 & 2,5 & 45,0 & 2,7 \\
\hline $30-25$ & 53,2 & 2,4 & 47,2 & 2,2 & 53,3 & 2,7 & 48,9 & 2,2 & 51,2 & 2,5 \\
\hline $25-20$ & 59,1 & 2,4 & 54,4 & 2,2 & 60,1 & 2,7 & 54,8 & 2,2 & 57,5 & 2,5 \\
\hline $20-15$ & 65,0 & 2,4 & 61,5 & 2,2 & 66,8 & 2,7 & 60,7 & 2,2 & 63,8 & 2,5 \\
\hline $15-10$ & 70,8 & 2,4 & 68,7 & 2,2 & 73,6 & 2,7 & 66,5 & 2,2 & 70,1 & 2,5 \\
\hline $10-05$ & 70,8 & 2,4 & 68,7 & 2,2 & 73,6 & 2,7 & 66,5 & 2,2 & 70,1 & 2,5 \\
\hline
\end{tabular}

Onde: $\mathrm{TBS}=$ temperatura de bulbo seco $\left({ }^{\circ} \mathrm{C}\right), \mathrm{PS}=$ potencial de secagem.

Não há informações disponíveis que permitam comparar os programas de secagem para as mesmas espécies, porém os valores obtidos neste trabalho são similares aos valores médios obtidos por Barbosa et al. (2005) para madeira de híbridos naturais de Eucalyptus. Dependendo da espécie, também são similares aos valores de temperatura e potencial de secagem obtidos por Andrade (2000) para E. grandis e E. tereticornis.

Santos (2002) recomenda para E. grandis temperaturas variando de $40{ }^{\circ} \mathrm{C}$ a $65^{\circ} \mathrm{C}$ e potenciais de secagem de 2,0 a 2,7. O programa utilizado por Batista et al. (2013) para secagem de E. grandis, E. saligna e E. dunnii, com temperatura inicial de $40{ }^{\circ} \mathrm{C}$, temperatura final de $66^{\circ} \mathrm{C}$ e potencial de secagem no ponto de saturação das fibras de 2, é simular aos definidos nesse trabalho para outras espécies. Da mesma forma, Travan et al. (2010) utilizaram temperaturas entre $45^{\circ} \mathrm{C}$ e $70{ }^{\circ} \mathrm{C}$ ao longo da secagem de E. grandis e E. trabutii. As temperaturas iniciais e finais recomendadas nesse trabalho para $E$. pilularis são cerca de $15^{\circ} \mathrm{C}$ abaixo das utilizadas por Cabardo \& Langrish (2006) para a mesma espécie.

Recomenda-se a validação desses programas de secagem através da avaliação da intensidade de defeitos antes e ao final do processo.

\section{Conclusão}

A secagem drástica permite a definição de programas de secagem para madeira de eucalipto de diferentes espécies, levando-se em consideração o comportamento durante a secagem.

As temperaturas iniciais recomendadas variam em função da espécie entre 40,0 e $47,3{ }^{\circ} \mathrm{C}$, o potencial de secagem entre 2,16 e 2,66 e a temperatura final entre 66,5 e $73,6^{\circ} \mathrm{C}$. Esses parâmetros não estão relacionados com a massa específica, reforçando a necessidade de um método que considere o comportamento durante a secagem. 
Foram identificados dois grupos distintos, sendo um que suporta condições de secagem mais severas, formado por Corymbia torelliana e E. grandis x E. urophylla e o outro que demanda condições mais suaves, formado por E. pilularis e E. cloeziana. A madeira de E. resinifera pode ser submetida à secagem nas condições definidas para quaisquer dos grupos.

\section{Referências}

ANDRADE, A. de. Indicação de programas para secagem convencional de madeiras, SP. 2000. 72 f. Dissertação (Mestrado em Ciências e Tecnologia da Madeira) - Escola Superior de Agricultura "Luiz de Queiroz", Piracicaba.

ASSIS, T. F. Aspectos do melhoramento de Eucalytpus para obtenção de produtos sólidos de madeira. In: WORKSHOP: TÉCNICAS DE ABATE, PROCESSAMENTO E UTILIZAÇÃO DA MADEIRA DE EUCALIPTO, 1999, Viçosa, MG, Anais... Viçosa, MG: SIF/ UFV, 1999. p. 61-72.

BARBOSA, C. G.; LIMA, J. T.; ROSADO, S. C. da S.TRUGILHO, P. F. Elaboração de programa de secagem para madeiras de clones de híbridos de Eucalyptus spp. Cerne, Lavras, v. 11, n. 1, p. 40-48, jan./mar. 2005.

BATISTA, D. C.; KLITZKE, R. J.; ROCHA, M. P.; MUÑIZ, G. I. B.; BATISTA, T. R. Volume loss as a tool to assess kiln drying of Eucalyptus wood. Floresta e Ambiente, Rio de Janeiro, v. 20, n. 2 , p. 250-256, 2013. DOI: 10.4322/floram.2013.017

BATISTA, D. C.; KLITZKE, R. J.; ROCHA, M. P. Qualidade da secagem convencional conjunta da madeira de clones de três espécies de Eucalyptus sp. Ciência Florestal, Santa Maria, RS, v. 25, n. 3, p. 711-719, 2015. DOI: 10.5902/1980509819621.

BRANDÃO, A. T. O.; JANKOWSKY, I. P. A screening to select kiln schedules. IPEF Internacional, Piracicaba, n. 2, p. 20-24, 1992.

CABARDO, S.; LANGRISH, T. Within-tree variability in the drying properties for blackbutt timber in New South Wales. Maderas: Ciencia y Tecnologia, n. 8, v. 1, p. 15-24, 2006.

COLODETTE, J. L.; GOMES, C. M.; GOMES, F. J.; CABRAL, C. $\mathrm{P}$. The Brazilian wood biomass supply and utilization focusing on eucalypt. Chemical and Biological Technologies in Agriculture, Portici, v. 1, n. 25, p. 1-8, 2014. DOI: 10.1186/s40538-014-0025-x.

DUCATTI, M. A.; JANKOWSKY, I. P.; ANDRADE, A. de. Condições operacionais da secagem convencional em indústrias madeireiras no município de Tietê, SP. Scientia Forestalis, Piracicaba, n. 59, p. 101-113, jun. 2001.

DURLO, M. A. Tecnologia da madeira: peso específico. Santa Maria, RS: Centro de Pesquisas Florestais, 1991. 29 p. (CEPEF. Série técnica, n. 8).

ELEOTÉRIO, J. R.; HORNBURG, K. F.; REICHERT, D.; BAGATTOLI, T. R.; MENEGHELLI, I. Efeito da espécie e da condição de secagem na formação de defeitos da madeira serrada de eucalipto. Scientia Forestalis, Piracicaba, v. 42, p. 41-47, 2014.
ELEOTÉRIO, J. R.; SILVA, C. M. K. Comparação de programas de secagem para Cumaru (Dipteryx odorata), Jatobá (Hymenaea spp) e Muiracatiara (Astronium lecointei) obtidos por diferentes métodos. Scientia Forestalis, Piracicaba, v. 40, p. 537-545, 2012.

ELEOTÉRIO, J. R.; SILVA, C. M. K. Programas de secagem para marupá (Simarouba amara), pará-pará (Jacaranda copaia) e virola (Virola surinamensis). Floresta, Curitiba, v. 44, p. 313-322, 2014. DOI: $10.5380 /$ rf.v44i2.30169

EUCALIPTO a madeira do futuro. Revista da Madeira, Curitiba, n. 59, p. 3-114. Edição especial.

GALVÃO, A. P. M.; JANKOWSKY, I. P. Secagem racional da madeira. São Paulo: Nobel, 1985. 111 p.

GORVARD, M. R.; ARGANBRIGHT, D. G. Development of kiln drying schedules severity indices for degrade control - Part I: theoretical development. Wood Science and Technology, New York, n. 13, p. 137-152, 1979.

JANKOWSKY, I. P. Metodologia simplificada para indicação de programas de secagem. 2009. 129 f. Tese (Livre-docência) - Escola Superior de Agricultura "Luiz de Queiroz", Piracicaba.

JANKOWSKY, I. P.; SANTOS, G. R. V. dos; ANDRADE, A. de. Secagem da madeira serrada de eucalipto. Piracicaba, n. 199, dez. 2003. (IPEF. Circular técnica, n. 199).

LONGUE JÚNIOR, D.; COLODETTE, J. L. Importância e versatilidade da madeira de eucalipto para a indústria de base florestal. Pesquisa Florestal Brasileira, Colombo, v. 33, n. 76, p. 429-438, 2013. DOI: 10.4336/2013.pfb.33.76.528.

MARTINS, V. A. Secagem de madeira serrada. Brasília, DF: IBDF, 1988. 56 p.

OLIVEIRA, J. T. S.; HELLMEISTER, J. C.; TOMAZELLO FILHO, M. Variação do teor de umidade e da densidade básica na madeira de sete espécies de eucalipto. Revista Árvore, Viçosa, MG, v. 29, n. 1, p. 115-127, 2005. DOI: 10.1590/S0100-67622005000100013.

REDMAN, A. L.; BAILlERES, H.; TURNER, I.; PERRÉ, P. Mass transfer properties (permeability and mass diffusivity) of four Australian hardwood species. Bioresources, Raleigh, n. 7, v. 3, p. 3410-3424, 2012.

SANTOS, G. R. V. dos. Otimização da secagem da madeira de Eucalyptus grandis [Hill ex Maiden]. 2002. 81 f. Dissertação (Mestrado) - Escola Superior de Agricultura "Luiz de Queiroz" Universidade de São Paulo, Piracicaba.

SEVERO, E. T. D. Qualidade da secagem de madeira serrada de Eucalyptus dunnii. Ciência Florestal, Santa Maria, RS, v. 10, n. 1, p. 109-124, 2000.

SIMPSON, W. T. Method to estimate dry-kiln schedules and species groupings: tropical and temperate hardwoods. Madison: Department of Agriculture, Forest Service, Forest Products Laboratory, 1996. $55 \mathrm{p}$.

SIMPSON, W. T.; VERRILL, S. P. Estimating kiln schedules for tropical and temperate hardwoods using specific gravity. Forest Products Journal, Madison, v. 47, n. 7/8, p. 64-68, 1997. 
TRAVAN, L.; ALLEGRETTI, O.; NEGRI, M. Eucalyptus drying process: qualitative comparison of different clones cultivated in Italy. In: CONFERENCE COST ACTION E53 “THE FUTURE OF
QUALITY CONTROL FOR WOOD \& WOOD PRODUCTS", 1. 2010, Edinburgh. Proceedings... Edinburgh: Cost, 2010. p. 1 - 14. Disponível em: <http:/www.coste53.net/downloads/Edinburgh/ Edinburgh-Presentation/43.pdf>. Acesso em: 10 maio 2014. 
